\title{
Une Nouvelle station d'Ophioglossum lusitanicum L. en Oranie (Algerie Occidentale).
}

\section{Boumediene Medjahdi ${ }^{1 *}$, Assia Letreuch-Belrouci ${ }^{1}$ et Ibrahim Babali ${ }^{2}$}

'Laboratoire de Gestion Conservatoire de l'eau, du Sol et des Forêts et Développement durable des zones montagneuses de la région de Tlemcen. Université de Tlemcen.

2Laboratoire d'écologie et gestion des écoystèmes naturels, département de biologie et environnement. Université de Tlemcen

\section{Corresponding author}

B. Medjahdi

e-mail: zoinif@yahoo.fr

Recibido: 23 octubre 2017

Aceptado: 2 noviembre 2017

Publicado on-line: diciembre 2017
New station of Ophioglossum lusitanicum L. in Oranie (Western of Algeria)

Mots clés: Ophioglossum lusitanicum, ptéridophytes, Région de Tlemcen, Ouest algérien

Key words: Ophioglossum lusitanicum, ptéridophyte, region of Tlemcen, the algerian West
Les ptéridophytes constituent le groupe le plus archaïque des végétaux supérieurs de la planète. C'est aussi un groupe largement moins diversifié que les spermaphytes. En Algérie se groupe n'est représenté que par 58 taxons dont un endémique (Dobignard et Chatelain, 2010). La plus grande partie de ces taxons se trouvent inféodés aux formations forestières des régions centre et Est du nord du pays, alors qu'en Oranie ce nombre est très réduit à cause de la sécheresse et de la forte dégradation des milieux favorables à leur développement (Medjahdi et al., 2013). La plupart des taxons se trouvent ainsi dans un état alarment de disparitions (Meddour, 2007). L'Ophioglossum lusitanicum L. n'échappe pas à cette réalité.

La famille d'Ophioglossaceae n'est représentée que par un seul genre en Algérie, le genre Ophioglossum L. Ce dernier comporte deux espèces, l'O. vulgatum $\mathrm{L}$. qui est une espèce rarissime selon Quézel et Santa (1962) et l'O. lusitanicum. espèce aussi très localisée observée que dans quelques stations au nord du pays.

\section{Chorologie d'Ophioglossum Iusitanicum}

Cette petite ptéridophyte de quelques centimètres de haut $(2$ à $7 \mathrm{~cm}$ ) difficilement observable sur le terrain à cause de sa taille et son écologie, se trouve généralement mêlée à des plantes de plus grandes tailles (Medjahdi et al., 2013). Ce taxon de distribution méditerraneo- macaronésien n'est présent en Afrique du nord qu'au Maroc, l'Algérie et la Tunisie. (Salvo \& Escamez, 1989).

En Algérie l'Ophioglossum lusitanicum ne se trouve que dans 9 stations dont 5 au centre du pays et 3 en Oranie (Casas et Sànchez, 2002). Dans la région de Tlemcen une seule station a été observée jusqu'à nos jours (Santa, 1949) (tab. 1 et fig. 1).

Nous décrivant dans ce travail une nouvelle station d'Ophioglossum lusitanicum (fig. 2). Cette dernière est située dans la forêt d'lfry à $10 \mathrm{~km}$ au

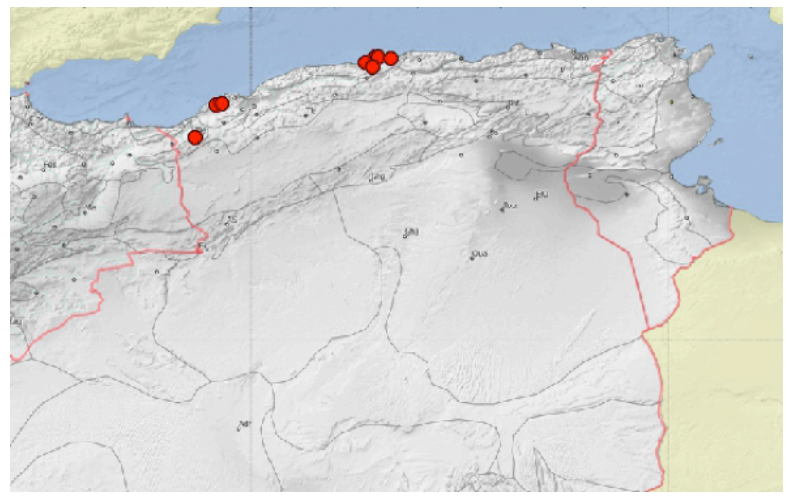

Figure 1: Représentation des stations d'Ophioglossum lusitanicum en Algèrie. Figure 1. Representation of the stations of Ophioglossum lusitanicum in Algèrie 


\begin{tabular}{|c|c|c|}
\hline Région & station & source \\
\hline Alger & $\begin{array}{l}\text { au sommet du rocher Charlemagne, forêt } \\
\text { de Baïnem, près d'Alger. Terrains rocail- } \\
\text { leux }\end{array}$ & Faurel s/n, 10-XI-1943 (MPU-A. Dubuis) \\
\hline Alger & environs d'Alger: Bouzaréa. Pelouses & Durando s/n, 1861 (MPU-Afrique N) \\
\hline Alger & forêt de Réghaïa. Clairières & A. Dubuis s/n, 04-XI-1934 (MPU-A. Dubuis) \\
\hline Alger & $\begin{array}{l}\text { la Réghaïa, } 50 \text { m. Clairières des forêts } \\
\text { sablonneuses de Quercus suber }\end{array}$ & $\begin{array}{l}\text { R. C. J. E. Maire s/n, } \\
\text { 30-XII-1928 (MA 466248; MPU-Afrique N). }\end{array}$ \\
\hline Blida & Bou-Ismaël & Clauson s/n, I/II (MPU-Afrique N). \\
\hline Blida & Boufarik, hippodrome de Xila. Pelouses & Duvernay s/n, 26-III-1920 (MPU-Afrique N). \\
\hline Oran & $\begin{array}{l}\text { plateau du versant gauche du ravin de } \\
\text { Noiseux aux abords du chemin } \\
\text { forestière }\end{array}$ & Doumergue s/n, I-1919 (MPU-Afrique N). \\
\hline Oran & Djebel Murdjadjo à Oran & G. Munby 68, III-1850 (MPU-Afrique N). \\
\hline Tlemcen & $\begin{array}{l}\text { supra urbem Tlemcen, 900-1000 m. In } \\
\text { pascuis arenaceis et calvitiis silvarum } \\
\text { Quercus suberis }\end{array}$ & $\begin{array}{l}\text { R. C. J. E. Maire } s / n, 19-I I I-1923 \text { (MPU- } \\
\text { Afrique N). }\end{array}$ \\
\hline
\end{tabular}

Tableau 1. Répartition de l'Ophioglossum lusitanicum en Algérie d'après Casas et Sànchez (2002). Table 1. Distribution of Ophioglossum lusitanicum in Algeria according to Casas et Sànchez (2002).

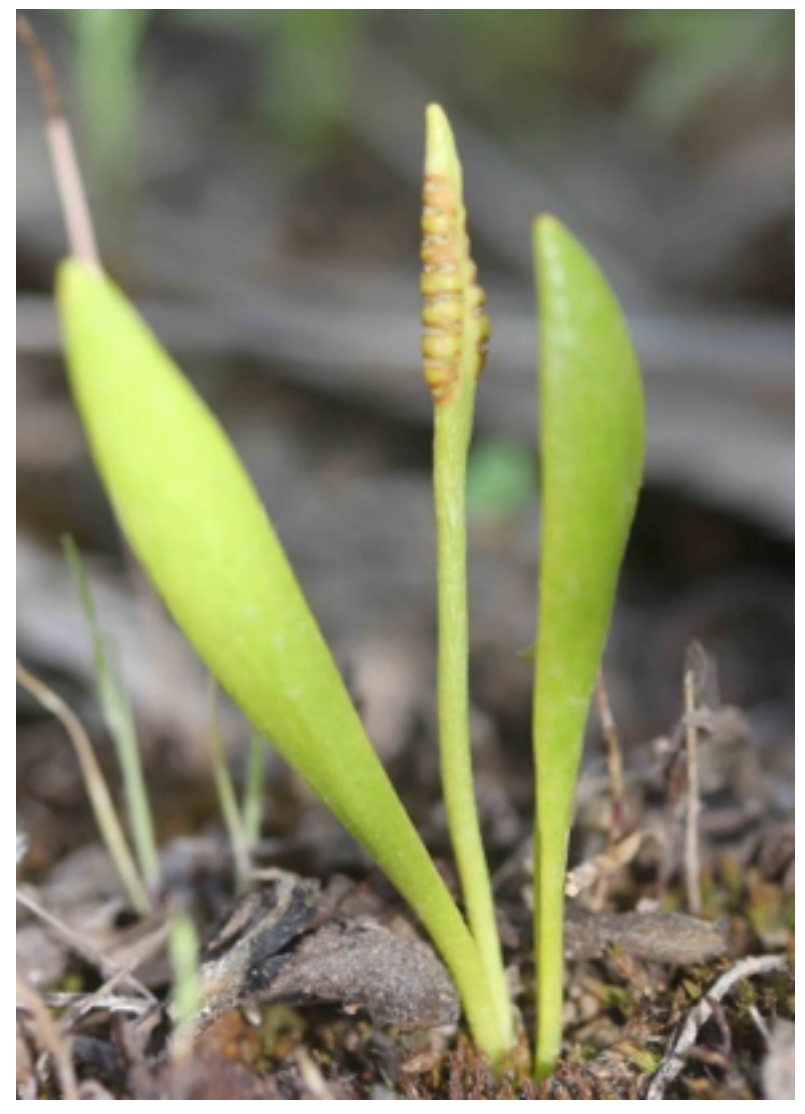

Figure 2: Ophioglossum lusitanicum L. forêt d'Ifry. Figure 2. Ophioglossum lusitanicum L. Forest of Ifry
Nord-Est de la ville de Tlemcen. Tout comme la première (décrite dans la région de Tlemcen dans la forêt de Zarifet par Munby citée par Maire 1952), notre station se trouve dans une subéraie.

Nous avons trouvé l'espèce en question dans une pelouse sous un buisson de Cistus monspeliensis $\mathrm{L}$. dans une clairière de la subéraie. La végétation présente sur $1 \mathrm{~m}^{2}$ à côté de notre ptéridophyte est présentée par les espèces suivantes: Isoetes durieui Bory, Gagea chabertii A. Terracc., Tuberaria inconspicua (Pers.) Willk., Trifolium campestreSchreb., Trifolium angustifolium L., Ornithopus compressus L., Bellis annua subsp. microcephala (Lange) Nyman, Ranunculus paludosus Poir et Rumex bucephalophorus L. subsp. bucephalophorus. Cette pelouse est située au point GPS 34.916083, -1.202200, sur un sol sablonneux profond à $950 \mathrm{~m}$ d'altitude. $\mathrm{La}$ proximité d'un suinte permet au sol de garder une humidité suffisante pour le développement de cette plante une bonne partie de l'année. La population est composée d'une dizaine d'individus concentrés sur $10 \mathrm{~m}^{2}$.

\section{Références}

Casas, F. J. \& Sánchez, A. J. (edit.) (2002). Asientos para un atlas corólogico de la flora occidental, 25 
Cavanillesia altera 2, Madrid, $818 p$

Dobignard, A. \& Chatelain, C. (2010). Index synonymique flore d'Afrique du Nord. Volume 1 : Ptéridophyta, Gymnospermae et Monocotyledoneae. Editions Des Conservatoire et Jardin Botaniques, Genève. 455 p.

Ferrero L.M., Medina, L., Baonza ,J. \& Aedo, C. (2016). Ophioglossum azoricum C. Presl y Elatine alsinastrum L. en la comunidad de Madrid. Acta Bot. Malacitana 41. pp. $255-257$

Medjahdi, B., Letreuch-Belarouci, A., Prelly, R. (2013). Actualisation du catalogue des pteridophytes du Nord-ouest algerien (Region de Tlemcen). Acta Bot. Malacitana. 38, pp 33-39

Meddour, R. (2007). Taxinomie, chorologie et régression desptéridophytes d'Algérie : synthèse bibliographique. Articles Réseaux Télabotanica. p15

Maire, R. (1952). Flore de l'Afrique du Nord. Volume 1. Ed. Le chevalier Paris. d'Alger. Paris. 146p

Quèzel, P. \& Santa, S. (1962). Nouvelle flore de l'Algérie et des régions désertiques méridionales. CNRS, Paris, Tome 1. $558 \mathrm{p}$.

Salvo, A.E. \& Escamez, A.M. (1989). Análisis biogeográfico y ecológico de la pteridoflora del Macizo del Gurugú (NE de Marruecos). Anales Jard. Bot. Madrid 46 (2) Madrid (España). pp.593-598.

Santa, S. -1949 - Catalogue des plantes de l'Algérie occidentale et du Maroc oriental. Extrait de B.S.G.A. de la Provence d'Oran. T.72, Fascicule 226, 128p. 